\section{Response to "Commentary on identity of fibroblast pneumocyte factor: rat vs. human"}

Advance online publication, 31 May 2017; doi:10.1038/pr.2017.86

To the Editor: Ballard's commentary (1) on our recent review of FPF is appreciated, particularly for the important issues that it raises. The basic discussion of FPF has always centered on whether FPF studies would ultimately identify an intrinsic factor with greater clinical utility to enhance fetal lung maturation than prenatal glucocorticoid therapy. Smith's publications repeatedly emphasized this goal. Ballard makes three crucial points regarding this goal: that the human fetus does not produce FPF, that fetal human type 2 cells develop surfactant synthesis without a need for fibroblast-type 2 cell communication, and that the timing of response of human fetal type 2 cells in culture to glucocorticoid stimulation is not consistent with cell-cell communication invoked by FPF. He also uses new data from RNA deep sequencing of fetal human type 2 cells to indicate that none of the growth factors discussed in our review are consistent with FPF. Here we respond briefly to these points.

It is true that most fibroblast pneumocyte factor (FPF) studies have used rat or mouse fetal lung fibroblasts and type 2 cells. However, Smith was intensely interested in the generalizability of FPF, reporting its presence in rat, mouse, rabbit, and two species of monkey (2). Importantly, he identified FPF in humans. Using a strategy for partial purification adopted from his original approach with fetal rat fibroblast-conditioned media (FCM), Smith showed that human amniotic fluid from a gestational period of 27 to 31 weeks contained significant FPF activity. The activity decreased in amniotic fluid from later gestations and disappeared as term gestation approached (3). Rat and mouse fetal lung FCM also exhibit a gestational regulation of FPF at corresponding stages $(4,5)$.

Ballard has contributed significantly to our understanding of fetal type 2 cell development by developing a method to isolate and culture pure fetal human type 2 cells from the second trimester. Smith, using a different approach, created a monoclonal human fetal type 2 cell line from a 31-week-old anencephalic fetus (6). Both Ballard's cells and Smith's cells exhibited the ability to differentiate into mature surfactantexpressing cells in culture. Ballard makes the point that his cells did so without apparent fibroblast input; however, we suggest that this is not quite accurate. Careful examination of both Smith's and Ballard's purification protocols shows that the fetal type 2 cells were cultured with their associated fetal lung fibroblasts for at least $24 \mathrm{~h}$ before final type 2 cell purification procedures were carried out. It is possible that this exposure in vitro to fibroblasts affected the subsequent behavior of the purified type 2 cell cultures. We appreciate Ballard's caveat regarding the proportion of fibroblasts present. No study has systematically evaluated fibroblast:type 2 cell ratios in the production of FPF. In addition, as we understand Ballard's method, the pure type 2 cells were routinely cultured with budesonide, a glucocorticoid. It is not clear whether the fetal type 2 cells were primed to develop spontaneous maturation by the fibroblast and budesonide exposures.

Ballard has elegantly demonstrated the timing of SP-B gene expression in human fetal type 2 cells in response to glucocorticoid stimulation. We summarized the effects of the FPF candidates on surfactant protein production; however, there are no time-response studies using FCM or these candidates to compare with the large body of work developed by Ballard. This is a clear gap in the fibroblast-type 2 cell model that should be addressed.

The expression data described by Ballard are indeed interesting and are likely to lead to important new areas of study. However, they do not eliminate any of the putative FPF candidates discussed in our review. For example, Ballard states that the signal for neuregulin-1beta (NRG) was unchanged. However, NRG is produced as a transmembrane protein that must be cleaved to release the active component into the extracellular space. Other, possibly unknown, processing mechanisms may be important for each factor we reviewed. Thus, a response time need not account for new protein synthesis.

As Ballard points out, determining whether FPF is involved in human fetal lung type 2 cell development is important for an understanding of the regulation of all surfactant components. In this respect, it is unfortunate that, as Smith's work with FPF progressed, he and others came to focus on rat and mouse culture models and not on human cells. We re-emphasize that the purpose of our article was to review the available evidence that may support the conclusion that one or more of KGF, leptin, or NRG is the long-sought FPF, and not to identify one of these factors as FPF. That said, we do feel that the total evidence indicates that FPF activity is present and active in the human fetal lung. The studies by Smith and Ballard in human fetal type 2 cells produced some common conclusions, but also conclusions in apparent disagreement. We feel that the evidence indicates that FPF activity is present and is actively involved in the development of surfactant production in human fetal lung type 2 cells; however, clearly more information is needed. Seeking 
an understanding of fundamental mechanisms when experimental results diverge is one of the aspects that make science research exciting.

\section{STATEMENT OF FINANCIAL SUPPORT}

This study was supported by NIH NHLBI HL085648.

Disclosure: The authors declare no conflict of interest.

Heber C. Nielsen ${ }^{\mathbf{1}}$, George King ${ }^{\mathbf{2}}$ and Max H. Cake ${ }^{2}$

${ }^{1}$ Department of Pediatrics, Graduate Program in Cell and Molecular Developmental Biology, Tufts Medical School, Boston, Massachusetts; ${ }^{2}$ School of Veterinary and Life Sciences, Murdoch University, Perth, Western Australia, Australia.

Correspondence: Heber C. Nielsen (heber.nielsen@tufts.edu)

\section{REFERENCES}

1. Ballard, PL. Commentary on identity of fibroblast pneumocyte factor: Rat versus human. Pediatric Research 2017;82: (this issue).

2. Smith BT. Lack of species specificity in production of fibroblastpneumonocyte factor by perinatal lung fibroblasts. In: Minkowski A, Monset-Couchard M, eds. Physiologic and Biochemical Basis for Perinatal Medicine. Basel, Switzerland: Karger, 1981: 54-8.

3. Seybold WD, Smith BT. Human amniotic fluid contains fibroblastpneumonocyte factor. Early Hum Dev 1980;4:337-45.

4. Caniggia I, Tseu I, Han RNN, Smith BT, Tanswell K, Post M. Spatial and temporal differences in fibroblast behavior in fetal rat lung. Am J of Physiol Lung Cell Mol Physiol 1991;261:L424-33.

5. Nielsen HC. Epidermal growth factor influences the developmental clock regulating maturation of the fetal lung fibroblast. Biochim Biophys Acta 1989;1012:201-6.

6. Tanswell AK, Smith BT. Cultured pulmonary epithelial cells: clonal isolation of human fetal alveolar type II cells. Birth Defects Orig Artic Ser 1980;16:249-59. 